\title{
Social intelligence: How to integrate research? A mechanistic perspective
}

\author{
Marcin Miłkowski ${ }^{1}$
}

Received: 27 April 2016 / Accepted: 18 November 2017 / Published online: 26 December 2017

(c) The Author(s) 2017. This article is an open access publication

\begin{abstract}
Is there a field of social intelligence? Many various disciplines approach the subject and it may only seem natural to suppose that different fields of study aim at explaining different phenomena; in other words, there is no special field of study of social intelligence. In this paper, I argue for an opposite claim. Namely, there is a way to integrate research on social intelligence, as long as one accepts the mechanistic account to explanation. Mechanistic integration of different explanations, however, comes at a cost: mechanism requires explanatory models to be fairly complete and realistic, and this does not seem to be the case for many models concerning social intelligence, especially models of economical behavior. Such models need either be made more realistic, or they would not count as contributing to the same field. I stress that the focus on integration does not lead to ruthless reductionism; on the contrary, mechanistic explanations are best understood as explanatorily pluralistic.
\end{abstract}

Keywords Integration $\cdot$ Unification $\cdot$ Mechanism $\cdot$ Integrated models

\section{From disunity of science to integrated models}

Social sciences, and in particular, research on social intelligence, is today highly fragmented and different disciplines are sometimes highly disconnected from each other. Although some theorists of social sciences find this situation commendable, I do not. For one, there is a danger of duplicating effort in different disciplines. For another, high-level abstract explanations in social sciences, when not properly constrained, or deepened (Strevens 2008), remain superficial and explanatorily weak. In particular, they run a danger of positing entities that play no causal role, if descriptions are not at all constrained by lower-level evidence.

To this, one may reply that such deepening is desired in physical or biological sciences, as these are mostly observable, while social sciences are "constructed."1 I disagree. All sciences, and in particular, social and cognitive sciences, are highly theoretical (Hohol 2013), positing multiple nonobservable entities for explanatory purposes. Mere observation is rarely ever explanatory. Similar point applies to

Marcin Miłkowski

mmilkows@ifispan.waw.pl

1 Institute of Philosophy and Sociology, Polish Academy of Sciences, ul. Nowy Świat 72, Warsaw, Poland "social construction": even if one agrees that some social sciences are busy with normative questions, their job is not merely do describe the social norms but also to explain why there are social norms at all, why they are considered binding by members of social institutions, and so on. Prescriptions, or normative guidance-such as the one offered by normative views on human rationality - are not explanatory in themselves, at least not obviously so. Moreover, contemporary cognitive sciences are very sensitive to social aspects of cognition, and that fact makes them relevant for any student of social intelligence, radically constructivist or not. Hence, I think there is sufficient reason to conclude that unifying social explanations is a worthwhile effort.

Even if the traditional question of unity of science seems to be answered today mostly in the negative (Fodor 1974; Dupré 1993), there is a related important question: What unifies a field of research? Why various studies are considered contributions to the same topic? An obvious answer, namely that it deals with the same entity or set of entities, seems to open a can of worms, especially in the light of various criticisms of the traditional unity of science. For example, one could say that one unifies cognitive sciences is the notion of cognition. Similarly, the field of social intelligence could be said to be unified because it deals, well, with social intelligence, not surprisingly. This is definitely a superficial

\footnotetext{
${ }^{1}$ I owe this observation to one of the reviewers of this paper.
} 
answer, and one that requires a substantial vindication of the theoretical notion at hand, be it cognition, social intelligence, or what not.

Moreover, John Dupré (1993) has argued that such intertheoretic identifications of entities are not straightforward. As he stresses, different biological disciplines idealize their entities in various ways. A lynx for ecological biology is an extremely idealized entity, especially when it comes to mathematical predator-prey models such as the Lotka and Volterra equation (for an analysis of the model, see Weisberg 2013). There is no role for genes, for example, in this equation, so as far as this model is concerned, lynx might have no genes. It just has to play the predator role. But for molecular biology, predation might have no importance at all, whereas genetic structure is crucial. And so on. Hence, different fields of study may focus simply on a different entity when they talk of social intelligence.

However, Dupré's conclusion that the lynx under study is a different entity in different fields is too quick. Even if particular models consider only some properties of an entity, the very applicability of the Lotka-Volterra model to a lynx presupposes a larger body of knowledge about the animal. Why is it a predator? Because it is a species of wild cats. This knowledge is necessary for the application of the predator-prey models but not contained therein. In other words, Dupré's argument fails, even if he is right that we may idealize lynx differently for different explanatory answers. Still, those different idealizations may be so disparate that they do not constitute a consistent field of research. Physics and theology can study the same entity, such as an ancient scripture; but they do not constitute the same field of research. In other words, there is little reason to think that the mention of the same entity, even if it is the same natural kind, makes different disciplines unified.

A disintegration of interdisciplinary research and the focus on very limited explanatory models has been one of the worries of Allen Newell and Herbert Simon, founding father of artificial intelligence and cognitive science. They both saw psychology as offering micro-theories, or theories of very limited scope, that did not contribute to a common view of the human mind (Newell 1973). Newell, as an alternative, proposed to unify the research program in cognitive science with a notion of a cognitive architecture (Newell 1990). In his opinion, a cognitive architecture can be used for creating multiple micro-theories and offers a unifying perspective on how the mind works. In a more contemporary context, researchers from the field of cognitive robotics suggested that unified cognitive-robotic architectures could be used to unify research efforts (Morse et al. 2011).

However, unified theories of cognition are not ways to unify fields of research. They may broaden the scope of theories but need not cross-fertilize the field as such, and, as Herbert Simon has stressed already in 1998 (Taatgen and
Anderson 2010), they stress the systems as a whole instead of mechanisms in the systems that make cognition possible. To theoretically integrate the whole field, there must be stress on such mechanisms. That is the lesson we take from this effort for the research on social intelligence: one way to unify, or at least integrate it is to develop mechanistic explanations.

In this paper, I develop a mechanistic account of unification and integration of the field of social intelligence, close in spirit to Simon's view. In Sect. 2, I introduce the mechanistic account of explanation as related to the question of integration different models and theories. Then, in Sect. 3, I tentatively sketch the mechanisms that can be studied to gain insight into social intelligence. In Sect. 4, I will apply this sketch to a case study of a new cognitive artifact, E-Memory. I then conclude by stressing that the mechanistic perspective supports explanatory pluralism.

The aim of the paper is to develop a theoretical understanding of integration in the domain of social intelligence rather than to offer new empirical insights or deepen the previous understanding of empirical phenomena. This is why the case study will be used only as an illustration of how one can analyze integrated explanations from the mechanistic point of view and to show that a genuine integration requires much more studies than are usually performed. Alas, the missing studies cannot be offered here. After all, the author of this paper is just another philosopher doing his work in the confines of his armchair.

\section{Mechanistic integration of research}

According to the received view on the unity of science, the goal of the unification is to create a single, universal theory. Hence, in this view, the most important relationships are inter-theoretical, reduction being the most prominent. However, it is neither realistic nor desirable to build a single theory in fields dealing with complex phenomena (Levins 1966; Wimsatt 2001, 2007; Weisberg 2007): Building multiple independent, usually highly idealized, models of phenomena may be much more useful than replacing them prematurely with a single theory. The core of the argument is that more robust results can be expected when they are produced independently by multiple statistically independent models than when they are generated with just one of them, as long as they draw from the same evidence base. Moreover, by testing how multiple theories or models match available evidence, researchers can compensate their confirmation bias, which makes all people prone to making a mistake of premature discarding alternative hypotheses (Farrell and Lewandowsky 2010). Additionally, for sciences other than fundamental physics, it is simply neither realistic nor practical to reduce 
them to a fundamental physical theory, such as quantum mechanics. In brief, there are genuine advantages in having multiple theories dealing with the same phenomena to be explained.

This consideration implies that integrating the discipline is not to be confused with unification, which is replacing all other theories with a single one, as long as alternative sound theories can be found. For our purposes, it means that the account of mechanistic integration will not aim at disposing with multiple models or theories. So how should such integration proceed?

Social intelligence clearly relies on multiple mechanisms. To skillfully interact with other agents, the agents needs to perceive them, which also requires sensory perception and intermodal transfer; one needs to understand agents as agents with their beliefs and desires (which is called 'mindreading'), which may also engage episodic memory, and long-term potentiation, and so on, and so on. Because of the multiplicity of mechanisms studied on different time and spatial scales, one could appeal to a notion of "vertical integration":

The natural sciences are already mutually consistent: the laws of chemistry are compatible with the laws of physics, even though they are not reducible to them. Similarly, the theory of natural selection cannot, even in principle, be expressed solely in terms of the laws of physics and chemistry, yet it is compatible with those laws. A conceptually integrated theory is one framed so that it is compatible with data and theory from other relevant fields. Chemists do not propose theories that violate the elementary physics principle of the conservation of energy: Instead, they use the principle to make sound inferences about chemical processes. A compatibility principle is so taken for granted in the natural sciences that it is rarely articulated, although generally applied; the natural sciences are understood to be continuous (Barkow et al. 1992).

Barkow et al. complain that in behavioral and social sciences such is not the case. These fields proceed in splendid mutual isolation. But how can one exactly understand this vertical integration, or compatibility of sciences?

One recent proposal of a general account of inter-theoretic and inter-model relationship is to use the notion of a constraint (Danks 2014). The weakest kind of constraint is a truth-constraint: two bodies of knowledge satisfy a truthconstraint just in case they can be both true at the same time. The notion of truth-constraint can then be used to precisely spell out the notion of vertical integration: namely, two bodies of knowledge are vertically integrated if and only if one body of knowledge truth-constraints the other and the first body cannot (in some weak sense) be false. (Notice that this definition accounts for vertical integration in a somewhat deflationary manner. The relationship is asymmetric in such a case, while normally, truth-constraining is horizontal, i.e., no body of knowledge is presupposed to be definitely true or more reliable than the other).

However, truth-constraining is a weak relation of logical coherence. The wave theory of light does not exclude the particle theory of light, so they satisfy the (horizontal) truth-constraint, even if they propose a completely different account of the basic nature of light. One stronger constraint concerns the nature of entities and processes (activities) presupposed by both theories. Both theories of light no longer satisfy such a constraint, unless a unifying theory is proposed: one that holds that light has both the nature of a particle and a wave at the same time.

The entity and process constraints can be easily applied to the mechanistic account of explanation, which is particularly sensitive to issues of interfield research (Darden and Maull 1977; Craver and Darden 2013). Before I go on to elucidate how that applies to the study of social intelligence, the notion of mechanistic explanation has to be made clear. According to new mechanism, to explain a phenomenon $\varphi$ is to elucidate the causal structure of the mechanism that gives rise to $\varphi$. While mechanisms are defined variously, the core idea is that they are organized systems, comprising causally relevant component parts and operations (or activities) thereof (for a recent review, see, e.g., Illari and Williamson 2011). Component parts of the mechanism interact, and their organized operation contributes to the capacity of the mechanism to exhibit $\varphi$.

Another important notion to be elucidated is the one of the interfield theory. The interfield theories are ones that relate at least two fields of study. By a field of study, Darden understands for example cytology or genetics rather than biology; in other words, it has a more restricted scope than a theory or a discipline. Two fields may appeal to the same spatiotemporal locations, entities or activities, and one of them may provide a better understanding of the spatiotemporal relationships, causal relationships, physical nature, structure or function thereof. In the case of social intelligence, it is quite clear that social relationships may be elucidated in various ways by various disciplines, from evolutionary and ecological biology (Meloni 2014) to cultural studies.

There are at least three ways fields may become integrated mechanistically: by simple integration, when the models of mechanisms can be considered pieces of puzzle that fit together; by interlevel relationship, when another level of organization is added to make explanation more complete; and by intertemporal integration (Craver and Darden 2013). In the case of simple integration, two fields may simply study social intelligence in a similar way but with a slightly different stress. For example, sociology of science studies researchers in a lab, and so does cultural anthropology, while the first field may use more quantitative 
analyses and historical evidence than the other to study similar phenomena.

The interlevel relationship is much more complex, as it may be confused with reduction. The need to introduce multiple levels of explanation is related to the nature of the mechanism under study. As Herbert Simon (1996) argued, on theoretical grounds, complex systems are likely to be near-decomposable, or composed of subsystems whose interactions are weak but not negligible. One facet of neardecomposability is hierarchical organization, in which different levels can be discerned, with interactions at a different order of magnitude. Such systems, as long as their capacities to be explained are identified, can be subject to mechanistic constitutive explanation, in which lower levels of organization explain higher levels. Levels are understood spatiotemporally; and the relationship between them is proper partwhole relationship (Craver 2007) (for a longer account, see Wimsatt 2007; for a more deflationary one; Eronen 2015). Note that the existence of a lower level explanation does not make the higher level disposable in this account of explanation: the higher level is explained by the interaction of the components and activities on the lower level, and the lower level contributes to the capacity of the higher level. In such a case, one could say that our knowledge of the higher level has been deepened, which also leads more empirical credentials to our previous beliefs about the higher level (Thagard 2007).

As long as explanations are integrated in an interlevel fashion, they are not only truth-constrained. The model of the lower level of a mechanism elucidates the activities and entities of the mechanism on the higher level. Such mechanistic explanations, called constitutive, cover at least three levels of organization: the bottom (-1) level, which is the lowest level in the given analysis and describes the internals of mechanism parts and their interactions; an isolated (0) level, at which the parts of the mechanism are specified along with their interactions (activities or operations); and the contextual $(+1)$ level, at which the function of the mechanism is seen in a broader context. Depending on the shared scientific practice, the bottom level in the explanation will vary (Machamer et al. 2000), as well as the upper levels. Note that one can easily introduce a further level if needed. Let us take an explanation that accounts for reproduction of bacteria. The reproduction of bacteria in a given environment (contextual level) is explained in terms of division (isolated level that ignores the environment), and division in terms of cellular mechanisms (the bottom level). The cellular-level mechanism can be further explained by its molecular parts, which would introduce a fourth level in this explanation.

The main, though fallible, heuristics in such explanations are localization and decomposition (Bechtel and Richardson 1993). For example, social capacities of a human being can be explained by a psychological model, the psychological capacities with a neuroscientific model, and many neuroscientific explanations proceed from neuroimaging studies (some of which are not reliable; see Trout 2008; Carp 2012). Note that from the mechanistic point of view, the model of, say, economical behavior of people involved in forex exchange, is explanatory only if it is complete. This means that the model needs to cite all relevant causal factors and clearly identify the explanandum phenomenon. In more concrete terms, it will mean that most micro-economical models will fail to be explanatory, as they tend to abstract away from crucial individual causal factors, in contrast to social models of behavior (Kuorikoski and Marchionni 2014). Also, many models that try to explain economical behavior only in neurophysiological terms fail to cite relevant factors known from psychological studies; and according to some, parts of neuroeconomics have lost touch with the rest of neuroscience by failing to integrate behavioral studies combined with physiological, pharmacological, or anatomical techniques that rely on animal models (Lauwereyns 2011).

The intertemporal integration applies to phenomena that can be analyzed, due to their hierarchical organization, on multiple temporal scales. For example, the behavior of a person interacting with a computer artifact may be explained by citing distal factors, relevant to explaining the history of computer artifacts that can be used by human beings. It can also be explained on a shorter time scale, by citing this person's skills and their acquisition. Also, it can be explained in a fine-grained fashion by using neuropsychological models of human-computer interaction. Different explanations of behavior will require researchers to appeal to mechanisms operating at different time scales.

The new mechanism frames the discovery of explanatorily relevant mechanisms for the mechanism under study in terms of looking up, down and around (Bechtel 2009). For example, Craver and Darden write:

One can look up to the higher-level mechanism of which it is a component. One can look back to the mechanisms that came before it or by which it developed. One can look forward to what comes after it. One can look around to see the even wider context within which it operates. The adequate explanation of many biological phenomena requires describing a temporally extended and multilevel mechanism. This is why many fields, working at multiple levels, often must integrate their work in the discovery of mechanisms (Craver and Darden 2013).

Let me summarize this section. By framing explanations of social intelligence in mechanistic terms, one can understand current scientific practice but also articulate certain norms of explanation, useful for integrating the field of social intelligence. In brief, the field will be integrated as 
long as it will study the complex mechanisms underlying social intelligence, and use knowledge about mechanisms operating at different levels of organization and various time scales to constrain hypotheses about the overall structure of the mechanism.

\section{Levels and time scales of the social mind}

In this section, I will tentatively sketch the mechanisms underlying social intelligence and cite relevant disciplines (for another similar proposal, see Castelfranchi 2014). Most abstractly, one can understand social intelligence as a capacity for skillful social interaction. Such abilities can be described on various levels of abstraction, for example in agent-based models (Squazzoni 2012; Conte and Paolucci 2014). Such models, however, usually only presuppose certain psychological capacities of agents, and for new mechanism, they require integration with cognitive science. They can, however, describe multi-agent cooperation and coordination. Quite obviously, social organization, with all kinds of business, military, power, and informal hierarchies, can be analyzed in terms of near-decomposable systems, and hence, idealized as mechanisms or components of larger mechanisms. But we can also look down to deepen the explanation of social mechanisms, and cognitive science has growing interest in the social.

Traditional cognitive science was methodologically individualist, and framed intelligent behavior in terms of processing of internal representations of individuals. Embodied and grounded cognition, the extended and scaffolded mind, enactivism and distributed cognition all challenge the traditional approach in different ways. Social intelligence is accounted for in terms of embodied interactions supported and extended by actively built cognitive niches. Despite the variety of approaches, they may be jointly dubbed "wide cognition"; they offer a new coherent picture of cognition, as well make it possible to integrate and unify interdisciplinary research. Below, I list how four approaches of wide cognition enable and require interfield integration. They are illustrated on Fig. 1, which depicts various aspects of skilful social interaction involved in airplane navigation [studied in much deeper detail as a distributed cognitive system by Hutchins (1995a)].

The claim of the embodied cognition (EC) is that the physical body of an agent is constitutively relevant for cognition; in other words, cognitive processing involves more than the brain. Core cognition, which essentially involves perception and action, depends deeply on the features of the physical body. This, obviously, means that biological mechanisms of human beings can become easily integrated on lower levels of the explanation. These biological mechanisms may include the features of our sensory and motor systems relevant for skillful action, including neural mechanisms of embodied joint attention (Sebanz et al. 2006).

The situated and embedded approach to cognition holds that cognition should be cashed out in terms of the interaction of the agent and its immediate surroundings. The extrabodily context constrains and enables cognition. For this reason, situated cognition needs to refer to behavioral studies as well as to basic biological and cognitive mechanisms of sociality, which includes such abilities as mindreading (Zawidzki 2013; Butterfill and Apperly 2013). Note that the mechanism of the social mind might not need to cite the controversial hypothesis of mirror neurons, sometimes assigned too many tasks without credible empirical evidence (Kilner and Lemon 2013; Hickok 2014).

The extended mind is the idea that cognitive processes are not necessarily brain-bound and can incorporate external resources such as tools, language, and external systems in order to enhance or augment cognitive processes. The difference of this approach from situated cognition is the emphasis that parts of what were traditionally considered the environment should properly be understood as part of the agent's mind. Even if the claim of the extended mind is exaggerated (Rupert 2009), this approach stresses the importance of material bases of cognition (Malafouris 2013). Here, cognitive archeology (Kirsh 2010) as well as the study of human-computer interactions (Dror 2007) becomes important.

The enactive approach to cognitive science recognizes a crucial inter-dependency between an autonomous agent and the world it inhabits. Cognitive activity is wholly defined neither by the agents nor their environment, but it emerges from their interaction. Again, enactive approaches can be easily linked with some biological disciplines and fields, one of which is biosemiotics (Favareau 2010). It is also very sensitive to temporal dimensions of human interactions on multiple scales (Silverman 2013).

These approaches may be illustrated with an example of airplane navigation (see Fig. 1), studied in depth by Hutchins (1995b). The whole plane cockpit with pilots constitutes a distributed cognitive system. Pilots physically (bodily) interact with different controls in the cockpit, which may be studied with the use of the embodied paradigm; but they are also embedded in the context of the cockpit, which is built so that it would appropriately constrain their cognition and attention if necessary. Some of the cognitive load may be off-loaded to specialized navigation instruments that extend the cognitive capacities of pilots. Moreover, if the pilots have a lot of joint experience, their interaction is more smooth; this aspect of cognitive processing is stressed by the enactive approach.

These approaches in cognitive science are immediately relevant for the study of social intelligence, and they do not deny the importance of the brain or individual mechanisms. But wide cognition does caution against adopting 


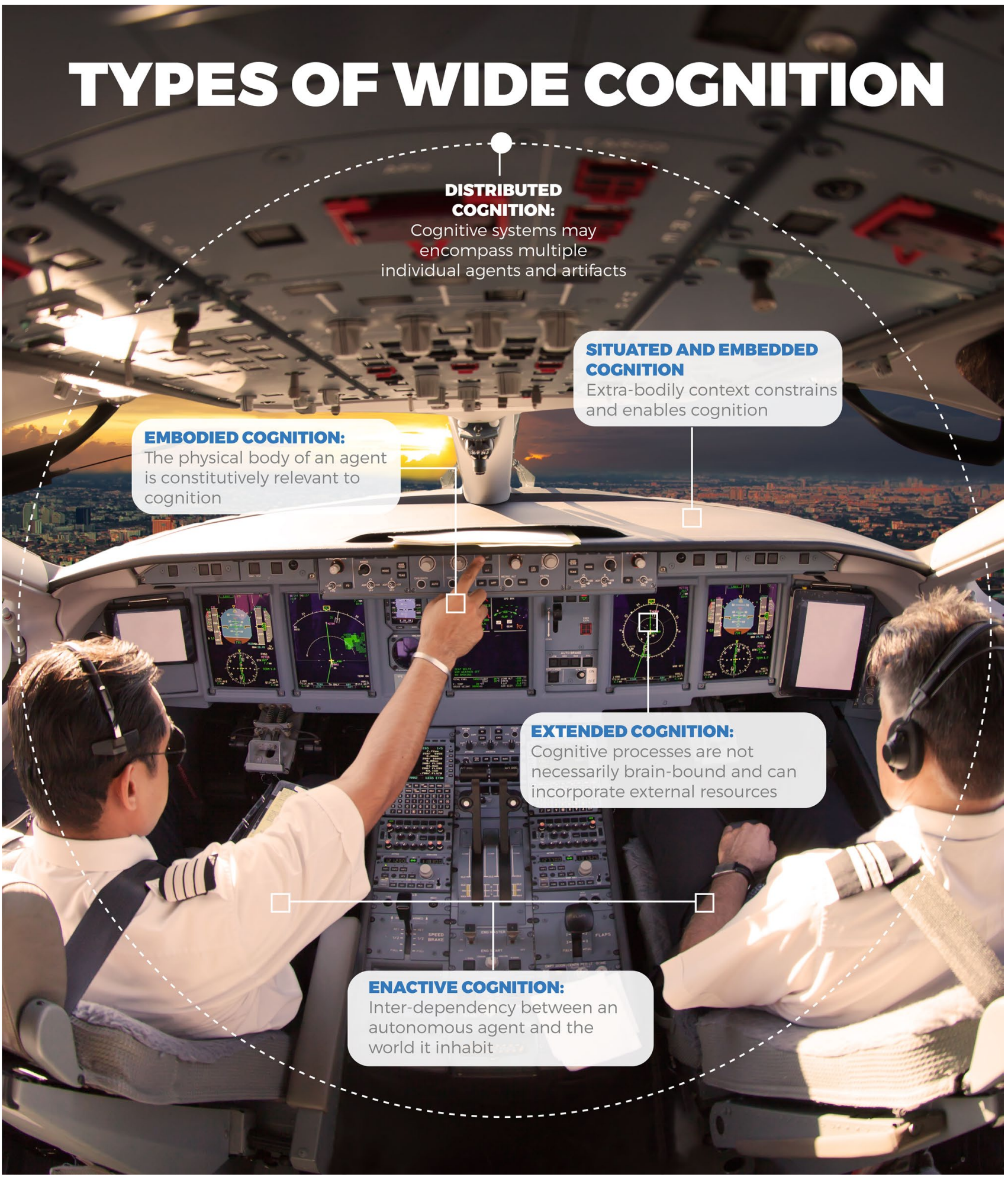

Fig. 1 Approaches to the study of social intelligence as applied to aircraftnavigation

an excessively narrow perspective that abstracts emotions away from the broader bodily, social, and cultural contexts that play a critical role in their development and functioning. Obviously, one factor critically important phenomenon for study of social intelligence is language, which is understood in current cognitive science not as mere realization of a formal grammar but also as involving multiple levels of cultural interaction and coordination, as well as internal 
mechanisms, also involving bodily interactions (Pecher and Zwan 2005; Cowley 2009; Glenberg 2010; Pezzulo et al. 2011). I suggest that different approaches of wide cognition offer multiple constraints on social theorizing; at least, these are truth-constraints, but ideally, we should strive at identifying common mechanisms that enable distributed, embodied and embedded cognitive processing.

At the same time, there are also competing explanations of various phenomena of social intelligence. Should one frame linguistic conventions in propositional and game-theoretic terms (Lewis 1969)? Or maybe it requires an evolutionary point of view (Skyrms 2010)? Or a view that mentions institutional contexts (Tummolini and Castelfranchi 2006), and temporal dynamics (Raczaszek-Leonardi and Kelso 2008)? Not all these views can be true at the same time, and integration is not just a matter of conceptual investigation. One could also see these different approaches as competing idealizations; however, they cannot be treated easily as supporting multiple-model idealization (Weisberg 2007) as they do not offer predictions or explanations for the same set of phenomena (if philosophical accounts of convention can offer any predictions for empirical phenomena, for that matter). However, one can see a set of common mechanisms for solving coordination problems in many of those approaches; sketching those is definitely beside the scope of this paper, whose aim is to defend a certain programmatic attitude to the study of social intelligence.

Another challenge remains to specify how to investigate cultural and social phenomena so as to include them all in unified models of social and cultural cognition. For example, so called network goods have value to someone only if other interactive parties also have them. It makes no sense to own a fax machine if nobody else owns one. This phenomenon can be studied on different time scales, and it is an open question how to integrate economical analyses with the psychological research on joint action, and the ethnographic studies typical of distributed cognition with computational modeling in evolutionary game theory, as it is also an example of a co-adaptive behavior.

\section{Case study: E-Memory}

In this section, the account of explanation introduced in previous sections will be applied to one case study, which is E-Memory. E-Memory is a new cognitive artifact that effectively enhances human capacities for remembering, by being similar to natural (biological) memory; it is defined by Clowes (2013) as "a heterogeneous bunch of devices and systems which fulfill similar functions either by replacement, extension or augmentation". In psychological terms, E-Memory systems are explicit, long-term storage systems. Think of sharing photos of everyday activities on social networks and using those as a kind of memory, by tagging them with dates, names of people and places. Clowes stresses that electronic media support characteristic features of E-Memory. These are:

Totality E-Memory is supposed to totally record all activities with high fidelity.

Practical cognitive incorporability E-Memory can be customized and used on electronic devices (for example, to remember locations of jars in the kitchen or phone numbers).

Autonomy E-Memory storage is processed usually in the cloud; other users can tag photos or movies on their own. Media can be processed in the cloud using machine learning systems to detect known or familiar faces, quite independently from the fact if the person who made the photo can recognize a face or not.

Entanglement Just because other users can interact with E-Memory systems of other users, the content of E-Memory may become entangled, tracking interactions between individuals, groups etc.

Clowes argues that because of these features, one may successfully apply the extended mind framework to E-Memory: the user's mind is extended, or constantly supported and augmented by electronic devices that promise the total recall of the past. Interestingly, these devices may be used by people with various memory deficits. At the same time, a proper study of the effects of E-Memory cannot neglect the way users using this technology deal with their environments and other people, as E-Memory systems can be social (and in an entangled way). In other words, the study of E-Memory should include not only the perspective of an individual user, but also of other people interacting with him, as E-Memory augments social interactions. Take for example a study of SenseCam, a wearable camera used as a pictorial diary to improve autobiographical memory in Mrs $\mathrm{B}$, a patient with limbic encephalitis (Berry et al. 2007). $\mathrm{Mr}$ B, the husband of Mrs B, was part of the inquiry: they both watched the images captured automatically by the camera to review them. It turned out that both Mrs and Mr B preferred SenseCam to a written diary, even if SenseCam would also capture boring and mundane tasks, and not only salient, more memorable events. However, the great deal of effort put in having a written diary was not rewarding as compared to the effortless capture of images using SenseCam.

What is however missing from this (preliminary) study is the assessment of long-term effects of SenseCam. These include not only neurological consequences but also broader social interactions. It would be also worthwhile seeing how much social tagging of images might help (or not) Mrs B to quickly find salient events (rather than the boring ones). What is also unknown is the influence of SenseCam on the bodily interactions of Mrs B. Even if SenseCam is mounted on the head of the patient and produces a movie from the point of view of 
the wearer, it may influence her own behavior (she might be moving more slowly to avoid blurry images, for example). After all, she has an autobiographical memory deficit but she might be currently aware of the fact that she is wearing a camera. In other words, wearing a camera might influence her bodily posture and movements, and potentially discourage from sport activities.

Most probably, the social interactions with other people may be highly influenced by the look of the camera. After all, all interactions will be recorded, so no offence or even a white lie might go unnoticed at the end of the day. This obviously raises questions regarding the privacy of interactions with people wearing SenseCams. E-Memory is easily accessible to multiple users, in contrast to biological memory. People interacting with disabled people who wear SenseCam may behave as if they were constantly eavesdropped. In other words, such technologies should be studied and understood also from the point of view of the social consequences of wider adoption of similar technologies. Only then may we understand all costs, and not only obvious health benefits of E-Memory.

A full mechanistic model of SenseCam would need to include not only its operations and components in an individual user but also the structure of the environment with which the user interacts using SenseCam. An interesting open question is the level of organization on which the model should "bottom out": namely, how deep are the effects of SenseCam adoption for a user. If its use has long-term consequence for the user's brain (in virtue of neural plasticity), then obviously this has to be accounted for. Ditto for long-term effects on social interaction, which, in turn, may be highly influence by cultural practices that govern such norms as privacy of intimate interaction. In such a case, the mechanistic model of SenseCam has to be integrated with a model of cultural transmission [as it is already done, for example, in neuroanthropology (Lende and Downey 2012)].

To sum up, a complete study of E-Memory systems for social intelligence should not only involve the study of individuals using these systems but also their environment and other people. These studies may also uncover reasons why some new technologies, such as Google Glass, which seemed to offer a new and easy access to various kinds of information, actually are not quickly adopted by users. One hypothesis is that such technologies are much more disruptive for social interaction because of their immersive character than tablets or smartphones (even if they are not offering full virtual reality at all). Another is that they are network goods: social interaction could be enhanced, as it is via the broad use of social media on smartphones, if there were a sufficient number of other users interacting socially using such devices.

\section{Conclusion}

In this paper, I merely sketched a mechanistic perspective on the integration of the field of social intelligence. A short example was then described, namely E-Memory systems. A complete case study of examples how mechanistic explanations furnish researchers with multiple constraints in their theorizing would require much more space. Instead of suggesting that there is a single, privileged theory of social intelligence, to which all other theories or models should be reduced, new mechanism stresses that understanding complex phenomena requires rich, multilevel models operating at multiple time scales. As such, it can help establish common research topics and identify the core submechanisms of social intelligence.

The interlevel nature of constitutive mechanistic explanations makes new mechanism a natural ally of explanatory pluralism (Miłkowski 2013; Gervais 2014). Instead of suggesting that there should be just a bottom-level causal explanation of all levels of organization of a given complex system, new mechanism insists that explanations at all levels are needed. These different explanations need not belong to the same discipline. They may be shared among various fields and disciplines. In other words, constitutive mechanistic explanations do not require different disciplines to become completely lumped together, as long as they can provide input for a common body of knowledge about a given mechanism.

This leads to a related issue. The assumption that the whole field of social intelligence is to be integrated may turn out premature. In principle, social intelligence may remain just a hub of interaction between different fields concerned with social and mental phenomena; it would not constitute a separate field then. However, the same principles would apply then: as long as we're interested in discovery of real mechanisms of social interaction of cognitive agents, there is a need to avoid excessive fragmentation of research, which may only need to isolation of subfields and duplication of effort.

Acknowledgements The author wishes to thank the anonymous reviewers of ECSI-2014 Conference and for AI and Society for their helpful suggestions. The work on this paper was financed by the Polish National Science Center grant under decision DEC-2014/14/E/ HS1/00803. A shorter version of this paper appeared in ECSI-2014 proceedings.

Open Access This article is distributed under the terms of the Creative Commons Attribution 4.0 International License (http://creativecommons.org/licenses/by/4.0/), which permits unrestricted use, distribution, and reproduction in any medium, provided you give appropriate credit to the original author(s) and the source, provide a link to the Creative Commons license, and indicate if changes were made. 


\section{References}

Barkow JH, Cosmides L, Tooby J (1992) The adapted mind. Evolutionary psychology and the generation of culture. Oxford University Press, New York and London

Bechtel W (2009) Looking down, around, and up: mechanistic explanation in psychology. Philos Psychol 22:543-564. https://doi. org/10.1080/09515080903238948

Bechtel W, Richardson RC (1993) Discovering complexity: Decomposition and localization as strategies in scientific research. Princeton University Press, Princeton

Berry E, Kapur N, Williams L et al (2007) The use of a wearable camera, SenseCam, as a pictorial diary to improve autobiographical memory in a patient with limbic encephalitis: a preliminary report. Neuropsychol Rehabil 17:582-601. https://doi. org/10.1080/09602010601029780

Butterfill SA, Apperly IA (2013) How to construct a minimal theory of mind. Mind Lang 28:606-637. https://doi.org/10.1111/ mila. 12036

Carp J (2012) The secret lives of experiments: methods reporting in the fMRI literature. Neuroimage 63:289-300. https://doi. org/10.1016/j.neuroimage.2012.07.004

Castelfranchi C (2014) For a science of layered mechanisms: beyond laws, statistics, and correlations. Front Psychol 5:536. https:// doi.org/10.3389/fpsyg.2014.00536

Clowes RW (2013) The cognitive integration of E-Memory. Rev Philos Psychol 4:107-133. https://doi.org/10.1007/ s13164-013-0130-y

Conte R, Paolucci M (2014) On agent-based modeling and computational social science. Front Psychol 5:668. https://doi.org/10.3389/ fpsyg.2014.00668

Craver CF (2007) Explaining the Brain. Mechanisms and the mosaic unity of neuroscience. Oxford University Press, Oxford

Craver CF, Darden L (2013) In search of mechanisms: discoveries across the life sciences

Danks D (2014) Unifying the mind: cognitive representations as graphical models. MIT Press, Cambridge

Darden L, Maull N (1977) Interfield theories. Philos Sci 44:43-64

Dror IE (2007) Cognitive technologies and the pragmatics of cognition. John Benjamins Pub., Amsterdam; Philadelphia

Dupré J (1993) The disorder of things: metaphysical foundations of the disunity of science. Harvard University Press, Cambridge Mass

Eronen MI (2015) Levels of organization: a deflationary account. Biol Philos 30:39-58. https://doi.org/10.1007/s10539-014-9461-z

Farrell S, Lewandowsky S (2010) Computational models as aids to better reasoning in psychology. Curr Dir Psychol Sci 19:329-335. https://doi.org/10.1177/0963721410386677

Favareau D (2010) Essential readings in biosemiotics. Springer, Netherlands, Dordrecht

Fodor JA (1974) Special sciences (or: the disunity of science as a working hypothesis). Synthese 28:97-115. https://doi.org/10.1007/ BF00485230

Gervais R (2014) A framework for inter-level explanations: outlines for a new explanatory pluralism. Stud Hist Philos Sci Part A 48:1-9. https://doi.org/10.1016/j.shpsa.2014.07.002

Glenberg AM (2010) Embodiment as a unifying perspective for psychology. Wiley Interdiscip Rev Cogn Sci 586-596. https://doi. org/10.1002/wcs. 55

Hickok G (2014) The myth of mirror neurons: the real neuroscience of communication and cognition. WW Norton, New York

Hohol M (2013) Wyjaśnić umysł: struktura teorii neurokognitywnych. Copernicus Center Press, Kraków

Hutchins E (1995a) Cognition in the wild. MIT Press, Cambridge

Hutchins E (1995b) How a Cockpit remembers its speeds. Cogn Sci 19:265-288. https://doi.org/10.1207/s15516709cog1903_1
Illari PM, Williamson J (2011) What is a mechanism? Thinking about mechanisms across the sciences. Eur J Philos Sci 2:119-135. https://doi.org/10.1007/s13194-011-0038-2

Kilner JM, Lemon RN (2013) What we know currently about mirror neurons. Curr Biol 23:R1057-R1062. https://doi.org/10.1016/j. cub.2013.10.051

Kirsh D (2010) Explaining artifact evolution. In: Malafouris L, Renfrew C (eds) Cognitive life of things. McDonald Institute for Archaeological Research, Cambridge, pp 121-142

Kuorikoski J, Marchionni C (2014) Unification and mechanistic detail as drivers of model construction: models of networks in economics and sociology. Stud Hist Philos Sci Part A 5-12. https://doi.org/10.1016/j.shpsa.2014.05.005

Lauwereyns J (2011) The anatomy of bias: how neural circuits weigh the options. MIT Press, Cambridge Mass, London

Lende DH, Downey G (eds) (2012) The encultured brain: an introduction to neuroanthropology. MIT Press, Cambridge Mass

Levins R (1966) The strategy of model building in population biology. Am Sci 54:421-431

Lewis D (1969) Convention: a philosophical study. Harvard University Press, Cambridge

Machamer P, Darden L, Craver CF (2000) Thinking about Mechanisms. Philos Sci 67:1-25

Malafouris L (2013) How things shape the mind: a theory of material engagement

Meloni M (2014) How biology became social, and what it means for social theory. Sociol Rev. https://doi. org/10.1111/1467-954X.12151

Miłkowski M (2013) Explaining the computational mind. MIT Press, Cambridge

Morse AF, Herrera C, Clowes R et al (2011) The role of robotic modelling in cognitive science. New Ideas Psychol 29:312-324. https://doi.org/10.1016/j.newideapsych.2011.02.001

Newell A (1973) You can't play 20 questions with nature and win: Projective comments on the papers of this symposium. In: Chase WG (ed) Visual information processing. Academic Press, New York, pp 283-308

Newell A (1990) Unified theories of cognition. Harvard University Press, Cambridge

Pecher D, Zwan RA (2005) Grounding cognition: the role of perception and action in memory, language. Cambridge University Press, Cambridge

Pezzulo G, Barsalou LW, Cangelosi A et al (2011) The mechanics of embodiment: a dialog on embodiment and computational modeling. Front Psychol 2:5. https://doi.org/10.3389/ fpsyg.2011.00005

Rączaszek-Leonardi J, Kelso JAS (2008) Reconciling symbolic and dynamic aspects of language: toward a dynamic psycholinguistics. New Ideas Psychol 26:193-207. https://doi.org/10.1016/j. newideapsych.2007.07.003

Rupert RD (2009) Cognitive systems and the extended mind. Oxford University Press, Oxford

Sebanz N, Bekkering H, Knoblich G (2006) Joint action: bodies and minds moving together. Trends Cogn Sci 10:70-76. https://doi. org/10.1016/j.tics.2005.12.009

Silverman D (2013) Sensorimotor enactivism and temporal experience. Adapt Behav 21:151-158. https://doi. org/10.1177/1059712313482802

Simon HA (1996) The sciences of the artificial. MIT Press, Cambridge

Cowley SJ (2009) Distributed language and dynamics. Pragmat Cogn 17:495-507. https://doi.org/10.1075/p\&c.17.3.01cow

Skyrms B (2010) Signals: evolution, learning, and information. Oxford University Press, Oxford

Squazzoni F (2012) Agent-based computational sociology. WileyBlackwell, Chichester 
Strevens M (2008) Depth: an account of scientific explanation. Harvard University Press, Cambridge Mass

Taatgen N, Anderson JR (2010) The past, present, and future of cognitive architectures. Top Cogn Sci 2:693-704. https://doi. org/10.1111/j.1756-8765.2009.01063.x

Thagard P (2007) Coherence, truth, and the development of scientific knowledge. Philos Sci 74:28-47

Trout JD (2008) Seduction without cause: uncovering explanatory neurophilia. Trends Cogn Sci 12:281-282. https://doi.org/10.1016/j. tics.2008.05.004

Tummolini L, Castelfranchi C (2006) The cognitive and behavioral mediation of institutions: towards an account of institutional actions. Cogn Syst Res 7:307-323. https://doi.org/10.1016/j. cogsys.2005.11.014
Weisberg M (2007) Forty years of "the strategy": levins on model building and idealization. Biol Philos 21:623-645. https://doi. org/10.1007/s10539-006-9051-9

Weisberg M (2013) Simulation and similarity: using models to understand the world. Oxford University Press, New York

Wimsatt WC (2001) Richard Levins as philosophical revolutionary. Biol Philos 16:103-108

Wimsatt WC (2007) Re-engineering philosophy for limited beings: piecewise approximations to reality. Harvard University Press, Cambridge Mass

Zawidzki T (2013) Mindshaping: a new framework for understanding human social cognition. MIT Press, Cambridge MA 